\title{
Evaluation of exposure-specific risks from two independent samples: A simulation study
}

\author{
William M Reichmann ${ }^{1,2^{*}}$, David Gagnon ${ }^{2,4}$, C Robert Horsburgh $^{3}$, Elena Losina ${ }^{1,2}$
}

\begin{abstract}
Background: Previous studies have proposed a simple product-based estimator for calculating exposure-specific risks (ESR), but the methodology has not been rigorously evaluated. The goal of our study was to evaluate the existing methodology for calculating the ESR, propose an improved point estimator, and propose variance estimates that will allow the calculation of confidence intervals (Cls).

Methods: We conducted a simulation study to test the performance of two estimators and their associated confidence intervals: 1) current (simple product-based estimator) and 2) proposed revision (revised product-based estimator). The first method for ESR estimation was based on multiplying a relative risk (RR) of disease given a certain exposure by an overall risk of disease. The second method, which is proposed in this paper, was based on estimates of the risk of disease in the unexposed. We then multiply the updated risk by the RR to get the revised product-based estimator. A log-based variance was calculated for both estimators. Also, a binomial-based variance was calculated for the revised product-based estimator. 95\% Cls were calculated based on these variance estimates. Accuracy of point estimators was evaluated by comparing observed relative bias (percent deviation from the true estimate). Interval estimators were evaluated by coverage probabilities and expected length of the $95 \% \mathrm{Cl}$, given coverage. We evaluated these estimators across a wide range of exposure probabilities, disease probabilities, relative risks, and sample sizes.
\end{abstract}

Results: We observed more bias and lower coverage probability when using the existing methodology. The revised product-based point estimator exhibited little observed relative bias (max: 4.0\%) compared to the simple product-based estimator (max: 93.9\%). Because the simple product-based estimator was biased, 95\% Cls around this estimate exhibited small coverage probabilities. The $95 \% \mathrm{Cl}$ around the revised product-based estimator from the log-based variance provided better coverage in most situations.

Conclusion: The currently accepted simple product-based method was only a reasonable approach when the exposure probability is small $(<0.05)$ and the RR is $\leq 3.0$. The revised product-based estimator provides much improved accuracy.

\section{Background}

Exposure-specific risk (ESR) is defined as the risk of disease (or any outcome) given a specific exposure (or subgroup). ESRs are useful to clinicians because it allows a much more meaningful way of explaining risk to patients. They are also useful to investigators who are looking to use ESRs for their own work, which may include publishing their own work or planning studies. In the absence of having access to the primary data or a

\footnotetext{
* Correspondence: wreichmann@partners.org

'Department of Orthopedic Surgery, Brigham and Women's Hospital, 75

Francis Street, Boston, MA 02115, USA

Full list of author information is available at the end of the article
}

reported estimate of the ESR in the literature, the ESR can be estimated from two independent samples if the investigator knows the overall risk of disease and the relative risk (RR) of disease given the exposure of interest. There have been a number of published studies where ESRs have been calculated from two independent samples by multiplying the overall risk of disease from one sample by the RR from a second independent sample $[1,2]$. Stewart et al. computed the ESR of hip fracture given certain exposures (prior fracture, family history of fracture, low body weight, and smoking) in persons over the age of 70 in the United Kingdom [2]. This study found that the ESR of hip fracture among those with all

\section{Biomed Central}


4 exposures was $8.9 \%$. This was done by multiplying an overall risk of hip fracture of $1.91 \%$ by a RR of 4.66 [2].

Horsburgh computed the ESR of tuberculosis for multiple risk factors, along with $95 \%$ confidence intervals (CIs). The upper (lower) bound of the 95\% CI for the ESR was calculated by multiplying the upper (lower) bound of the $95 \%$ CI for the overall risk by the upper (lower) bound of the 95\% CI for the RR [1]. While there has been some work addressing the multiplication of two binomial parameters [3], to the best of our knowledge, there are no methodological articles evaluating the properties of the simple product-based estimator that was used in the articles by Stewart et al and Horsburgh.

In this article we set to address three objectives. The first is to evaluate the properties of the simple productbased estimator of the ESR used by Stewart et al and Horsburgh. The second objective is to propose an estimate of the variance of the ESR, which can subsequently be used for calculating 95\% CIs. Lastly, we propose a revised product-based estimator and two variances estimates for the revised point estimator which are used to calculate $95 \%$ CIs.

\section{Methods}

\section{Overview}

We designed and implemented a simulation study to examine the properties of two different estimators of the ESR and their 95\% CIs. The two estimators we sought to evaluate (and their associated CIs) were a simple product-based estimator and revised product-based estimator. Point estimators were evaluated by calculating the observed relative bias. Their 95\% CIs were evaluated using coverage probabilities and expected length given coverage for a wide range of parameters, including exposure probability, probability of disease among the unexposed, the RR of disease given exposure, and the sample size.

For the purposes of this paper, D represents having disease, E represents having exposure, subscript one denotes that the quantity comes from sample one, and subscript two denotes that the quantity comes from sample two. More careful examination of the mathematics behind this simple product-based estimator clearly shows that the estimator is at best a crude approximation of the ESR.

$$
P_{1}(D) * R R_{2}=P_{1}(D) * \frac{P_{2}(D \mid E)}{P_{2}(D \mid \bar{E})} \neq P(D \mid E)=E S R
$$

To estimate the ESR, this formula needs an estimate of the risk of disease in the unexposed $\left(P_{1}(D \mid \bar{E})\right)$ rather than the estimate of the overall risk of disease $\left(P_{1}(D)\right)$.

\section{Simple Product-Based ESR}

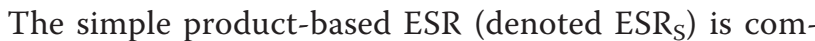
puted by simply multiplying the overall probability of disease from sample one by the RR from sample two. The formula is given below.

$$
\mathrm{ESR}_{\mathrm{S}}=\mathrm{P}_{1}(\mathrm{D}) * \mathrm{RR}_{2}=\mathrm{P}_{1}(\mathrm{D}) * \frac{\mathrm{P}_{2}(\mathrm{D} \mid \mathrm{E})}{\mathrm{P}_{2}(\mathrm{D} \mid \overline{\mathrm{E}})}
$$

\section{Variance and Confidence Interval for the Simple Product- Based ESR}

We first propose a formula for the variance of the simple product-based estimator using a natural log transformation. We assumed that the covariance between $\ln \left(\mathrm{P}_{1}\right.$ (D)) and $\ln \left(R_{2}\right)$ was zero because they are estimated from independent data sets.

$$
\begin{aligned}
& \operatorname{Var}\left(\ln \left(E_{S} R_{S}\right)\right)=\operatorname{Var}\left(\ln \left(P_{1}(D) * R_{2}\right)\right) \\
& =\operatorname{Var}\left(\ln \left(P_{1}(D)\right)+\ln \left(R_{2}\right)\right) \\
& =\operatorname{Var}\left(\operatorname { l n } \left(P_{1}(D)+\operatorname{Var}\left(\ln \left(R_{2}\right)\right)\right.\right. \\
& \quad+2 * \operatorname{Cov}\left(\ln \left(P_{1}(D)\right), \ln \left(R_{2}\right)\right) \\
& =\operatorname{Var}\left(\ln \left(P_{1}(D)\right)\right)+\operatorname{Var}\left(\ln \left(R_{2}\right)\right)
\end{aligned}
$$

(assuming independence)

$$
=\operatorname{Var}\left(\ln \left(\mathrm{P}_{1}(\mathrm{D})\right)\right)+\frac{1-\mathrm{P}_{2}(\mathrm{D} \mid \mathrm{E})}{\mathrm{P}_{2}(\mathrm{D} \mid \mathrm{E})^{*} \mathrm{n}_{2 \mathrm{E}}}+\frac{1-\mathrm{P}_{2}(\mathrm{D} \mid \overline{\mathrm{E}})}{\mathrm{P}_{2}(\mathrm{D} \mid \overline{\mathrm{E}})^{*} \mathrm{n}_{2 \overline{\mathrm{E}}}}
$$

To complete the formula for the variance of the natural log ESR we need the variance of the natural log of the overall risk $\left(\operatorname{Var}\left(\operatorname{In}\left(\mathrm{P}_{1}(\mathrm{D})\right)\right)\right)$. This is derived below using the delta method.

$$
\begin{aligned}
\operatorname{Var}\left(\ln \left(\mathrm{P}_{1}(\mathrm{D})\right)\right) & =\frac{1}{\mathrm{P}_{1}(\mathrm{D})^{2}} * \operatorname{Var}\left(\mathrm{P}_{1}(\mathrm{D})\right) \\
& =\frac{1}{\mathrm{P}_{1}(\mathrm{D})^{2}} * \frac{\mathrm{P}_{1}(\mathrm{D}) *\left(1-\mathrm{P}_{1}(\mathrm{D})\right)}{\mathrm{n}_{1}} \\
& =\frac{\left(1-\mathrm{P}_{1}(\mathrm{D})\right)}{\mathrm{n}_{1} * \mathrm{P}_{1}(\mathrm{D})}
\end{aligned}
$$

Substituting the result of equation 4 into equation 3 and we have the final variance for the natural log of the ESR:

$$
\begin{aligned}
& \operatorname{Var}\left(\ln \left(E_{S}\right)\right) \\
& =\frac{\left(1-P_{1}(D)\right)}{n_{1}{ }^{*} P_{1}(D)}+\frac{1-P_{2}(D \mid E)}{P_{2}(D \mid E)^{*} n_{2 E}}+\frac{1-P_{2}(D \mid \bar{E})}{P_{2}(D \mid \bar{E})^{*} n_{2 \bar{E}}}
\end{aligned}
$$

Because we tested our confidence intervals for sample sizes that were 250 or larger, the central limit theorem applies and we can use a normal approximation for 
estimating the $95 \%$ confidence interval.

$$
95 \% \mathrm{CI}=\exp \left(\ln \left(\mathrm{ESR}_{\mathrm{S}}\right) \pm 1.96 * \sqrt{\operatorname{Var}\left(\ln \left(\mathrm{ESR}_{\mathrm{S}}\right)\right)}\right)
$$

\section{Revised Product-Based ESR}

Note, from formula 1, we need an estimate of the risk of disease in the unexposed from sample $1\left(\mathrm{P}_{1}(\mathrm{D} \mid \overline{\mathrm{E}})\right)$, rather than the estimate of the overall risk of disease $\left(\mathrm{P}_{1}\right.$ (D)). Assuming that the risk of disease in the unexposed is not reported from sample 1 or sample 2, we can use the law of total probability to derive $P_{1}(D \mid \bar{E})$. By the law of total probability the following formula holds.

$$
\begin{aligned}
& P(D)=P(D \mid E) * P(E)+P(D \mid \bar{E}) * P(\bar{E}) \\
& =R R^{*} P(D \mid \bar{E}) * P(E)+P(D \mid \bar{E}) *(1-P(E)) \\
& =R R^{*} P(D \mid \bar{E}) * P(E)+P(D \mid \bar{E})-(P(D \mid \bar{E}) * P(E)) \\
& =(R R-1) * P(D \mid \bar{E}) * P(E)+P(D \mid \bar{E}) \\
& =P(D \mid \bar{E})^{*}[((R R-1) * P(E))+1]
\end{aligned}
$$

Next, solving for $\mathrm{P}(\mathrm{D} \mid \overline{\mathrm{E}})$ gives us:

$$
P(D \mid \bar{E})=\frac{P(D)}{\left[\left((R R-1)^{*} P(E)\right)+1\right]}
$$

Here, estimates of $\left.P_{1}(D)\right), P_{2}(E)$, and $R R_{2}$ are available in samples one and two as denoted by the subscripts. Then the final estimate for the revised product-based ESR is

$$
\mathrm{ESR}_{\mathrm{R}}=\frac{\mathrm{P}_{1}(\mathrm{D})}{\left[\left(\left(\mathrm{RR}_{2}-1\right)^{*} \mathrm{P}_{2}(\mathrm{E})\right)+1\right]} * \mathrm{RR}_{2} .
$$

\section{Variance and Confidence Interval for the Revised Product-}

\section{Based ESR}

The first estimate of variance derived for the revised product-based is derived for the natural log of the estimate. This is done similar to the derivation for the variance of the natural $\log$ of $\mathrm{ESR}_{\mathrm{s}}$ shown in equation 5 . The exception is that now we need to find variance of the natural $\log$ of the probability of disease among the unexposed in sample 1.

$$
\begin{aligned}
& \operatorname{Var}\left(\ln \left(\mathrm{ESR}_{\mathrm{R}}\right)\right)= \\
& \frac{\left(1-\mathrm{P}_{1}(\mathrm{D} \mid \overline{\mathrm{E}})\right)}{\mathrm{n}_{1}{ }^{*} \mathrm{P}_{1}(\mathrm{D} \mid \overline{\mathrm{E}})}+\frac{1-\mathrm{P}_{2}(\mathrm{D} \mid \mathrm{E})}{\mathrm{P}_{2}(\mathrm{D} \mid \mathrm{E})^{*} \mathrm{n}_{2 \mathrm{E}}}+\frac{1-\mathrm{P}_{2}(\mathrm{D} \mid \overline{\mathrm{E}})}{\mathrm{P}_{2}(\mathrm{D} \mid \overline{\mathrm{E}})^{*} \mathrm{n}_{2 \overline{\mathrm{E}}}}
\end{aligned}
$$

Thus a 95\% CI for $\mathrm{ESR}_{\mathrm{R}}$ can be constructed using the normal approximation shown in equation 6 by substituting $E_{S R_{R}}$ for $E S R_{S}$ and $\operatorname{Var}\left(\ln \left(E S R_{R}\right)\right)$ for $\operatorname{Var}\left(\ln \left(E S R_{S}\right)\right)$.
Since the ESR is a probability, the second estimate of the variance derived for $\mathrm{ESR}_{\mathrm{R}}$ is based on the binomial distribution. The variance for a binomial parameter $\mathrm{p}$ is $\frac{\mathrm{p}^{*}(1-\mathrm{p})}{\mathrm{n}}$. We chose to estimate the denominator of this formula by multiplying the sample size from sample 1 by the exposure probability from sample 2 . This provides a more conservative estimate of the variance because the denominator will be smaller. The final forms of this variance and the $95 \%$ CI using a normal approximation to the binomial distribution are shown below.

$$
\begin{aligned}
& \operatorname{Var}\left(\mathrm{ESR}_{\mathrm{R}}\right)=\frac{\mathrm{ESR}_{\mathrm{R}}{ }^{*}\left(1-\mathrm{ESR}_{\mathrm{R}}\right)}{\mathrm{n}_{1}{ }^{*} \mathrm{P}_{2}(\mathrm{E})} \\
& 95 \% \mathrm{CI}=\mathrm{ESR}_{\mathrm{R}} \pm 1.96 * \sqrt{\frac{\mathrm{ESR}_{\mathrm{R}}{ }^{*}\left(1-\mathrm{ESR}_{\mathrm{R}}\right)}{\mathrm{n}_{1}{ }^{*} \mathrm{P}_{2}(\mathrm{E})}}
\end{aligned}
$$

\section{Simulation study details}

All simulations and subsequent evaluations were performed using SAS statistical software, version 9.2 (SAS, Cary, NC). Populations of size 10 million were generated based on different exposure probabilities, probabilities of disease among the unexposed, and RRs of disease given the exposure. One thousand pairs of samples were drawn from the population to determine the sampling distribution of the overall probability of disease and the RR of disease given exposure. After the samples were generated, estimates of the RR and overall probability of disease (along with their 95\% CIs) were calculated for each sample.

For the purposes for this report we organize the results into four scenarios. Scenario 1 considers the situation where the exposure probability was low (.05) and the probability of disease among the unexposed was low (.02). Scenario 2 considers the situation where the exposure probability was low (.05) and the probability of disease among the unexposed was moderate (.09). Scenario 3 considers the situation where the exposure probability was high (.20) and the probability of disease among the unexposed was low (.02). Scenario 4 considers the situation where the exposure probability was high $(.20)$ and the probability of disease among the unexposed was moderate (.09). For all four scenarios we evaluated the properties of the two point estimators and three interval estimators across seven different RRs and four different sample size combinations (Table 1). We chose larger sample sizes because the probability of disease in the unexposed was low (.02) or moderate (.09) and investigators who perform this calculation would want to use the highest quality estimate available. Lastly, in order to evaluate properties of our estimates when 
Table 1 Parameters varied and all their possible values for the simulation study

\begin{tabular}{ll}
\hline Parameter & Possible values \\
\hline Exposure probability & $.05, .20$ \\
Probability of disease among unexposed & $.02, .09$ \\
RR & $1.0,1.5,2.0,2.5,3.0,4.0,5.0$ \\
Sample size combinations for the overall risk and $R R\left(N_{1} / N_{2}\right)$ & $250 / 250^{*}, 1,000 / 1,000,1,000 / 5,000,5,000 / 1,000,5,000 / 5,000$ \\
\hline
\end{tabular}

*Note: The $250 / 250$ sample size combination was only performed in Scenario 4 (probability of exposure $=.20$; probability of disease among unexposed $=.09$ ).

the sample size was small, we ran our simulations with a sample size of 250 for both sample 1 and sample 2 under the conditions of Scenario 4 only. We did not perform this analysis in the other three scenarios because the prevalence of exposure and disease among the unexposed was too small to provide a reliable estimate of the RR.

\section{Evaluation of ESR Estimators}

We calculated the estimated ESR using the simple product-based method and revised product-based method for each of the 1,000 pairs of samples. We evaluated the estimators using observed relative bias. Observed relative bias was defined as the difference between the average of the 1,000 estimates from the 1,000 pairs of samples and the assumed population ESR divided by the assumed population ESR. Observed relative bias can be described as the percent change from the true estimate.

\section{Evaluation of Confidence Intervals}

All 95\% CIs were evaluated using coverage probabilities. The coverage probability is defined as the probability that the interval covers the assumed population ESR. For each of the 1,000 pairs of samples we determine whether the assumed population ESR falls between the lower and upper bounds of the CI. The coverage probability is then determined by the number of times the interval covered divided by 1,000. Since we calculated 95\% CIs, we expect that our intervals would cover 950 times out of 1,000 (95\%).

Expected length given coverage was also evaluated for all of our 95\% CIs. For every 95\% CI that covered the true value of the ESR for a given pair of 1,000 samples, the length was calculated by subtracting the lower bound from the upper bound. We then calculated the average of these lengths to get the expected length given coverage. For example, if the coverage probability was $95.1 \%$ then 951 out of 1,000 intervals covered the true value of the ESR. Therefore the expected length given coverage is based on an $N$ of 951 . For the purpose of comparison, we also calculated the empirical 95\% CI and its length. This was done by examining the distribution of the direct estimator and taking the $2.5^{\text {th }}$ percentile to be the lower bound of the $95 \%$ CI and the $97.5^{\text {th }}$ percentile to be the upper bound of the $95 \% \mathrm{CI}$. The length of the empirical $95 \%$ CI was calculated by subtracting the $2.5^{\text {th }}$ percentile from the $97.5^{\text {th }}$ percentile.

\section{Case Study}

We tested our methodology using a case study in which we calculated the risk of symptomatic knee osteoarthritis (OA) in obese persons by age groups. The overall risk of symptomatic knee OA by age group was derived from Oliveria et al [4]. This article reports on one of the largest population-based studies that estimates the risk of symptomatic knee OA with a cohort of more than 130,000 members of a community health plan. The relative risk of symptomatic knee OA for obese persons (1.91) and proportion obese (0.371) was derived from Niu et al [5]. This study provides one of the most current estimates of the relative risk of symptomatic knee OA by obesity status and also had a substantial sample size $(\mathrm{N}=2,660)$. Since the study by Niu and colleagues only studied those ages 50-79, we limited our analysis to those ages 50-59, 60-69, and 70-79.

\section{Results}

Scenario 1: Low exposure probability (.05)/Low disease probability among unexposed (.02)

In the case where the probability of exposure was low (.05) and the probability of disease among the unexposed was low (.02), ESR $\mathrm{R}_{\mathrm{R}}$ performed better than $\mathrm{ESR}_{\mathrm{S}}$ with respect to observed relative bias. When the RR was 1.0 , the observed relative bias was near 0 for both estimators. However, as the RR increased the observed relative bias of ESR $\mathrm{S}_{\mathrm{S}}$ increased. This increased to a high of $31.4 \%$ when the RR was 5.0 and both sample sizes were 1,000 . In the same situation, $\mathrm{ESR}_{\mathrm{R}}$ exhibited an observed relative bias of $3.4 \%$. In general, as the RR increased in magnitude so did the observed relative bias of $\mathrm{ESR}_{\mathrm{S}}$, while the observed relative bias of $\mathrm{ESR}_{\mathrm{R}}$ was not larger than $4.0 \%$ (Table 2).

Coverage probabilities for the $95 \% \mathrm{CI}$ of $\mathrm{ESR}_{\mathrm{S}}$ were at least $95 \%$ when the RR was 2.0 or less, regardless of the sample size combination. However, as the RR increased (and subsequently the observed relative bias), the coverage probabilities began to fall below 95\%. The coverage probability fell to $87.1 \%$ when the RR was 5.0 and both sample sizes were 5,000 (Table 3). Coverage probabilities for the $95 \% \mathrm{CI}$ for $\mathrm{ESR}_{\mathrm{R}}$ using a log-based variance were 
Table 2 Observed relative bias for the simple product-based estimator (ESRS) and the revised product-based estimator (ESRR)

\begin{tabular}{|c|c|c|c|c|}
\hline \multicolumn{5}{|c|}{ Low exposure probability $(.05) /$ Low disease probability in unexposed $(.02)$} \\
\hline \multirow[b]{2}{*}{ RR/ESR } & \multicolumn{2}{|c|}{$\mathrm{N}_{1}=1,000, \mathrm{~N}_{2}=1,000$} & \multicolumn{2}{|c|}{$\mathrm{N}_{1}=5,000, \mathrm{~N}_{2}=5,000$} \\
\hline & ESRS & $\mathrm{ESR}_{\mathrm{R}}$ & ESRS & $E S R_{R}$ \\
\hline $1.0 / .02$ & $9.5 \%$ & $3.9 \%$ & $-1.4 \%$ & $-2.3 \%$ \\
\hline 2.0/.04 & $7.8 \%$ & $-2.6 \%$ & $4.5 \%$ & $-1.4 \%$ \\
\hline $3.0 / .06$ & $18.0 \%$ & $1.8 \%$ & $12.2 \%$ & $0.8 \%$ \\
\hline 4.0/.08 & $21.1 \%$ & $0.0 \%$ & $17.6 \%$ & $1.1 \%$ \\
\hline 5.0/.10 & $31.4 \%$ & $3.4 \%$ & $22.6 \%$ & $1.0 \%$ \\
\hline
\end{tabular}

\begin{tabular}{|c|c|c|c|c|}
\hline \multicolumn{5}{|c|}{ Low exposure probability (.05)/Moderate disease probability in unexposed (.09) } \\
\hline & \multicolumn{2}{|c|}{$\mathrm{N}_{1}=1,000, \mathrm{~N}_{2}=1,000$} & \multicolumn{2}{|c|}{$\mathrm{N}_{1}=5,000, \mathrm{~N}_{2}=5,000$} \\
\hline RR/ESR & $\mathrm{ESR}_{S}$ & $\mathrm{ESR}_{\mathrm{R}}$ & $\mathrm{ESR}_{\mathrm{S}}$ & $E S R_{R}$ \\
\hline $1.0 / .09$ & $0.1 \%$ & $-0.9 \%$ & $0.9 \%$ & $0.6 \%$ \\
\hline 2.0/.18 & $6.1 \%$ & $0.1 \%$ & $5.5 \%$ & $0.3 \%$ \\
\hline $3.0 / .27$ & $9.2 \%$ & $-1.4 \%$ & $10.4 \%$ & $0.2 \%$ \\
\hline $4.0 / .36$ & $16.6 \%$ & $0.4 \%$ & $15.9 \%$ & $0.5 \%$ \\
\hline $5.0 / .45$ & $22.0 \%$ & $0.7 \%$ & $21.2 \%$ & $0.8 \%$ \\
\hline
\end{tabular}

\begin{tabular}{|c|c|c|c|c|}
\hline \multicolumn{5}{|c|}{ High exposure probability $(.20) /$ Low disease probability in unexposed $(.02)$} \\
\hline & \multicolumn{2}{|c|}{$\mathrm{N}_{1}=1,000, \mathrm{~N}_{2}=1,000$} & \multicolumn{2}{|c|}{$N_{1}=5,000, N_{2}=5,000$} \\
\hline RR/ESR & $\mathrm{ESR}_{S}$ & $\mathrm{ESR}_{\mathrm{R}}$ & $\mathrm{ESR}_{\mathrm{S}}$ & $\mathrm{ESR}_{\mathrm{R}}$ \\
\hline $1.0 / .02$ & $8.7 \%$ & $1.1 \%$ & $-0.2 \%$ & $-1.3 \%$ \\
\hline 2.0/.04 & $26.3 \%$ & $-1.4 \%$ & $21.4 \%$ & $-0.1 \%$ \\
\hline 3.0/.06 & $45.0 \%$ & $-1.8 \%$ & $41.7 \%$ & $0.1 \%$ \\
\hline 4.0/.08 & $73.3 \%$ & $1.2 \%$ & $61.6 \%$ & $0.1 \%$ \\
\hline $5.0 / .10$ & $93.9 \%$ & $0.3 \%$ & $82.5 \%$ & $0.0 \%$ \\
\hline
\end{tabular}

\begin{tabular}{|c|c|c|c|c|}
\hline \multicolumn{5}{|c|}{ High exposure probability (.20)/Moderate disease probability in unexposed (.09) } \\
\hline & \multicolumn{2}{|c|}{$\mathrm{N}_{1}=1,000, \mathrm{~N}_{2}=1,000$} & \multicolumn{2}{|c|}{$N_{1}=5,000, N_{2}=5,000$} \\
\hline RR/ESR & $\mathrm{ESR}_{S}$ & $\mathrm{ESR}_{\mathrm{R}}$ & ESRS & $\mathrm{ESR}_{\mathrm{R}}$ \\
\hline $1.0 / .09$ & $-0.8 \%$ & $-0.4 \%$ & $-1.1 \%$ & $-1.0 \%$ \\
\hline 2.0/.18 & $22.6 \%$ & $0.7 \%$ & $20.4 \%$ & $0.2 \%$ \\
\hline $3.0 / .27$ & $40.9 \%$ & $-0.2 \%$ & $40.3 \%$ & $0.0 \%$ \\
\hline $4.0 / .36$ & $63.6 \%$ & $0.7 \%$ & $60.6 \%$ & $0.1 \%$ \\
\hline $5.0 / .45$ & $82.6 \%$ & $0.2 \%$ & $81.0 \%$ & $0.2 \%$ \\
\hline
\end{tabular}

$\mathrm{N}_{1}$ is the sample the overall risk is derived from.

$\mathrm{N}_{2}$ is the sample the relative risk is derived from.

Relative Risk/Exposure-Specific Risk (RR/ESR) values are the hypothesized values.

above 95\% across all RRs for three of the four sample size combinations $\left(\mathrm{N}_{1}=1,000, \mathrm{~N}_{2}=1,000 ; \mathrm{N}_{1}=5,000\right.$, $\mathrm{N}_{2}=1,000$; and $\left.\mathrm{N}_{1}=5,000, \mathrm{~N}_{2}=5,000\right)$. When the sample size combination was 1,000 for the overall risk (sample 1) and 5,000 for the RR (sample 2) the 95\% CI for $E_{S R}$ using a log-based variance failed to attain $95 \%$ coverage for all RRs (see additional file 1). The exact opposite relationship was observed for the $95 \%$ CI of $E_{R}$ using a binomial variance. This interval only attained $95 \%$ coverage when the sample size combination was $1,000 / 5,000$. In fact, these coverage probabilities well exceeded 95\% with the smallest coverage probability being $98.9 \%$ when the RR was 1.0 (Table 4).

Figure 1 shows the expected lengths given coverage of all the 95\% CIs constructed by different RRs (1.0, 2.0, and 5.0 respectively) for the 5,000/5,000 $\left(\mathrm{N}_{1} / \mathrm{N}_{2}\right)$ sample size combination. The expected length of the empirical 95\% CI is also shown. The expected length given coverage is largest for the $95 \% \mathrm{CI}$ around $\mathrm{ESR}_{\mathrm{S}}$, and the binomial-based variance yielded $95 \%$ CIs around $E_{R} R_{R}$ with smaller lengths than the log-based variance $95 \%$ CIs in this scenario.

\section{Scenario 2: Low exposure probability (.05)/Moderate disease probability among unexposed (.09)}

Increasing the probability of disease among the unexposed from .02 to .09 while keeping the exposure probability set to .05 did not drastically change our results. The observed relative bias of ESR still increased as the magnitude of the RR increased. When the RR was 5.0, 
Table 3 Coverage probability for the $95 \%$ confidence interval of the simple product-based estimator (ESRS) and revised product-based estimator (ESRR) using a log-based variance

\begin{tabular}{|c|c|c|c|c|}
\hline \multicolumn{5}{|c|}{ Low exposure probability $(.05) /$ Low disease probability in unexposed $(.02)$} \\
\hline \multirow[b]{2}{*}{ RR/ESR } & \multicolumn{2}{|c|}{$\mathrm{N}_{1}=1,000, \mathrm{~N}_{2}=1,000$} & \multicolumn{2}{|c|}{$N_{1}=5,000, N_{2}=5,000$} \\
\hline & $\mathrm{ESR}_{S}$ & $\mathrm{ESR}_{\mathrm{R}}$ & ESRS & $E S R_{R}$ \\
\hline $1.0 / .02$ & 96.8 & 97.3 & 97.5 & 97.5 \\
\hline 2.0/.04 & 96.4 & 98.1 & 95.9 & 97.2 \\
\hline 3.0/.06 & 95.7 & 98.3 & 92.7 & 96.6 \\
\hline 4.0/.08 & 94.1 & 98.2 & 90.3 & 98.0 \\
\hline $5.0 / .10$ & 93.9 & 98.4 & 87.1 & 97.7 \\
\hline
\end{tabular}

\begin{tabular}{|c|c|c|c|c|}
\hline \multicolumn{5}{|c|}{ Low exposure probability $(.05) /$ Moderate disease probability in unexposed $(.09)$} \\
\hline & \multicolumn{2}{|c|}{$N_{1}=1,000, N_{2}=1,000$} & \multicolumn{2}{|c|}{$\mathrm{N}_{1}=5,000, \mathrm{~N}_{2}=5,000$} \\
\hline RR/ESR & $\mathrm{ESR}_{\mathrm{S}}$ & $\mathrm{ESR}_{\mathrm{R}}$ & $\mathrm{ESR}_{\mathrm{S}}$ & $E S R_{R}$ \\
\hline $1.0 / .09$ & 96.8 & 97.2 & 95.9 & 96.2 \\
\hline 2.0/.18 & 95.8 & 96.8 & 93.4 & 96.3 \\
\hline 3.0/.27 & 94.3 & 97.0 & 87.8 & 96.4 \\
\hline 4.0/.36 & 89.3 & 96.1 & 70.8 & 97.0 \\
\hline $5.0 / .45$ & 83.2 & 96.6 & 45.0 & 96.6 \\
\hline
\end{tabular}

\begin{tabular}{|c|c|c|c|c|}
\hline \multicolumn{5}{|c|}{ High exposure probability $(.20) /$ Low disease probability in unexposed $(.02)$} \\
\hline & \multicolumn{2}{|c|}{$N_{1}=1,000, N_{2}=1,000$} & \multicolumn{2}{|c|}{$N_{1}=5,000, N_{2}=5,000$} \\
\hline RR/ESR & $\mathrm{ESR}_{\mathrm{S}}$ & $\mathrm{ESR}_{\mathrm{R}}$ & $\mathrm{ESR}_{\mathrm{S}}$ & $\mathrm{ESR}_{\mathrm{R}}$ \\
\hline $1.0 / .02$ & 97.6 & 98.5 & 94.5 & 96.6 \\
\hline 2.0/.04 & 94.2 & 98.9 & 85.6 & 98.0 \\
\hline 3.0/.06 & 89.5 & 99.0 & 55.1 & 98.6 \\
\hline 4.0/.08 & 76.8 & 99.2 & 22.3 & 99.4 \\
\hline $5.0 / .10$ & 65.4 & 99.6 & 4.2 & 99.4 \\
\hline
\end{tabular}

\begin{tabular}{|c|c|c|c|c|}
\hline \multicolumn{5}{|c|}{ High exposure probability $(.20) /$ Moderate disease probability in unexposed $(.09)$} \\
\hline & \multicolumn{2}{|c|}{$N_{1}=1,000, N_{2}=1,000$} & \multicolumn{2}{|c|}{$N_{1}=5,000, N_{2}=5,000$} \\
\hline RR/ESR & $\mathrm{ESR}_{S}$ & $\mathrm{ESR}_{\mathrm{R}}$ & ESRS & $\mathrm{ESR}_{\mathrm{R}}$ \\
\hline $1.0 / .09$ & 94.7 & 96.8 & 94.7 & 96.5 \\
\hline 2.0/.18 & 85.4 & 98.5 & 51.1 & 98.0 \\
\hline $3.0 / .27$ & 55.9 & 98.5 & 1.8 & 98.6 \\
\hline $4.0 / .36$ & 17.6 & 99.4 & 0 & 99.4 \\
\hline $5.0 / .45$ & 2.9 & 99.5 & 0 & 99.4 \\
\hline
\end{tabular}

$\mathrm{N}_{1}$ is the sample the overall risk is derived from.

$\mathrm{N}_{2}$ is the sample the relative risk is derived from.

Relative Risk/Exposure-Specific Risk (RR/ESR) values are the hypothesized values.

Italics denote coverage probabilities that did not attain $95 \%$.

the observed relative bias of ESR $_{\mathrm{S}}$ was greater than $20 \%$ for all sample size combinations. The observed relative bias of ESR $R_{R}$ was close to zero for all combinations of RR and sample size (Table 2).

Coverage probabilities for the $95 \% \mathrm{CI}$ of ESR $\mathrm{ES}_{\mathrm{S}}$ were less than $95 \%$ in most cases. The coverage probabilities were adversely affected by the increasing magnitude of the RR with a minimum coverage probability of $45 \%$ attained when the RR was 5.0 and the sample size was 5,000 for both samples (Table 3). Similar to Scenario 1, coverage probabilities for the $95 \% \mathrm{CI}$ of $\mathrm{ESR}_{\mathrm{R}}$ using a log-based variance exhibited at least $95 \%$ coverage in all cases except when the sample size the overall risk was derived from was 1,000 and the sample size the RR was derived from was 5,000 (see additional file 1). The 95\% CI for $\mathrm{ESR}_{\mathrm{R}}$ using a binomial variance showed the exact opposite relationship. Regardless of the magnitude of the RR, the coverage probability of the $95 \%$ CI for $E_{R}$ using a binomial variance was greater than $99 \%$ when the sample size the overall risk was derived from was 1,000 and the sample size the RR was derived from was 5,000 (Table 4).

Figure 2 shows the expected lengths given coverage of the $95 \%$ CIs. The expected length given coverage increases for all the intervals as the magnitude of the $R R$ increases. The expected lengths of the $95 \%$ CIs of $E S R_{S}$ and ESR $R_{R}$ using a log-based variance are similar when the RR is small. However, as the RR increases in 
Table 4 Coverage probability of the $95 \%$ confidence interval for the revised product-based estimator (ESRR) using a binomial variance

\begin{tabular}{|c|c|c|c|c|c|c|c|}
\hline \multicolumn{8}{|c|}{ Low exposure probability (.05)/Low disease probability in unexposed $(.02)$} \\
\hline \multirow[b]{2}{*}{$\begin{array}{l}\text { Sample Size } \\
\left(N_{1} / N_{2}\right)\end{array}$} & \multicolumn{7}{|c|}{ RR/ESR } \\
\hline & $1.0 / .02$ & $1.5 / .03$ & 2.0/.04 & $2.5 / .05$ & 3.0/.06 & 4.0/.08 & $5.0 / .10$ \\
\hline $1,000 / 1,000$ & 66.1 & 78.1 & 83.2 & 87.1 & 89.6 & 88.9 & 90.0 \\
\hline $1,000 / 5,000$ & 98.9 & 99.3 & 99.6 & 99.7 & 99.9 & 99.5 & 99.5 \\
\hline $5,000 / 1,000$ & 54.4 & 60.6 & 61.2 & 62.3 & 63.5 & 64.9 & 65.0 \\
\hline $5,000 / 5,000$ & 90.0 & 92.8 & 92.8 & 93.7 & 92.4 & 94.4 & 94.8 \\
\hline \multicolumn{8}{|c|}{ Low exposure probability (.05)/Moderate disease probability in unexposed (.09) } \\
\hline & \multicolumn{7}{|c|}{$\mathrm{RR} / \mathrm{ESR}$} \\
\hline $\begin{array}{l}\text { Sample Size } \\
\left(N_{1} / N_{2}\right)\end{array}$ & 1.0/.09 & $1.5 / .14$ & $2.0 / .18$ & $2.5 / .23$ & $3.0 / .27$ & $4.0 / .36$ & $5.0 / .45$ \\
\hline $1,000 / 1,000$ & 90.4 & 91.6 & 93.2 & 92.4 & 92.2 & 92.0 & 91.5 \\
\hline $1,000 / 5,000$ & 99.9 & 100 & 100 & 100 & 99.9 & 99.7 & 99.3 \\
\hline $5,000 / 1,000$ & 62.8 & 63.8 & 63.9 & 63.7 & 62.1 & 61.6 & 62.7 \\
\hline $5,000 / 5,000$ & 93.8 & 93.4 & 94.2 & 93.5 & 93.7 & 93.6 & 91.6 \\
\hline
\end{tabular}

High exposure probability (.20)/Low disease probability in unexposed (.02)

\begin{tabular}{|c|c|c|c|c|c|c|c|}
\hline \multirow[b]{2}{*}{$\begin{array}{l}\text { Sample Size } \\
\left(N_{1} / N_{2}\right)\end{array}$} & \multicolumn{7}{|c|}{$\mathrm{RR} / \mathrm{ESR}$} \\
\hline & $1.0 / .02$ & $1.5 / .03$ & $2.0 / .04$ & $2.5 / .05$ & $3.0 / .06$ & $4.0 / .08$ & $5.0 / .10$ \\
\hline $1,000 / 1,000$ & 89.1 & 91.2 & 91.8 & 92.6 & 91.5 & 92.5 & 93.8 \\
\hline $1,000 / 5,000$ & 99.7 & 99.1 & 99.4 & 99.0 & 98.0 & 98.8 & 98.1 \\
\hline $5,000 / 1,000$ & 63.9 & 68.0 & 64.7 & 69.3 & 70.7 & 70.5 & 71.9 \\
\hline $5,000 / 5,000$ & 92.1 & 94.0 & 95.1 & 94.8 & 94.3 & 94.5 & 93.5 \\
\hline
\end{tabular}

High exposure probability (.20)/Moderate disease probability in unexposed (.09)

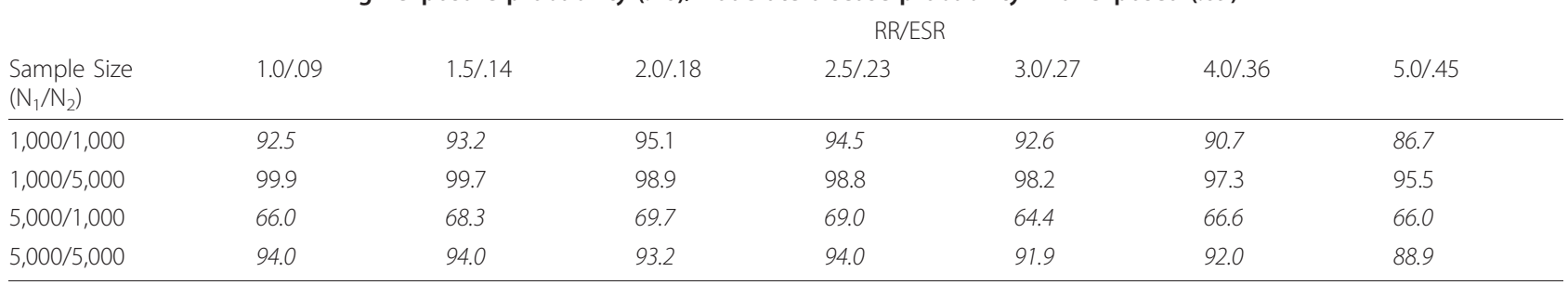

$\mathrm{N}_{1}$ is the sample the overall risk is derived from.

$\mathrm{N}_{2}$ is the sample the relative risk is derived from.

Relative Risk/Exposure-Specific Risk (RR/ESR) values are the hypothesized values.

Italics denote coverage probabilities that did not attain $95 \%$.

magnitude, the length of the $95 \% \mathrm{CI}$ for $\mathrm{ESR}_{\mathrm{S}}$ is greater than the length of the $95 \%$ CI for $\mathrm{ESR}_{\mathrm{R}}$ using a logbased variance.

\section{Scenario 3: High exposure probability (.20)/Low disease probability among unexposed (.02)}

Increasing the exposure probability from .05 to .20 while the probability of disease among the unexposed was .02 affected the results substantially for the existing methodology. The observed relative bias of $\mathrm{ESR}_{\mathrm{S}}$ was over $10 \%$ when the RR was 1.5 , over $20 \%$ when the $R R$ was 2.0 , and over $80 \%$ when the RR was 5.0. However, the observed relative bias of $E_{S R}$ was near $0 \%$ with the greatest observed relative bias being $-1.8 \%$ when the $R R$ was 3.0 and both sample sizes were 1,000 (Table 2).
In terms of coverage probability, the $95 \%$ CI for $\mathrm{ESR}_{\mathrm{S}}$ attained 95\% coverage only when the RR was small. When the RR was 5.0, the 95\% CI for ESR $\mathrm{S}$ had a coverage probability as low as $4.2 \%$ when the sample size was 5,000 for both samples. Similar to the previous two analyses, coverage probabilities for the $95 \%$ CI of $E S R_{R}$ using a log-based variance exhibited at least 95\% coverage in all cases except when the sample size the overall risk was derived from was 1,000 and the sample size the RR was derived from was 5,000 (see additional file 1 ). The $95 \%$ CI for $\mathrm{ESR}_{\mathrm{R}}$ using a binomial variance showed the exact opposite relationship. Regardless of the magnitude of the $R R$, the coverage probability of the $95 \% \mathrm{CI}$ for $E_{S R_{R}}$ using a binomial variance was greater than 99\% when the sample size the overall risk was derived 


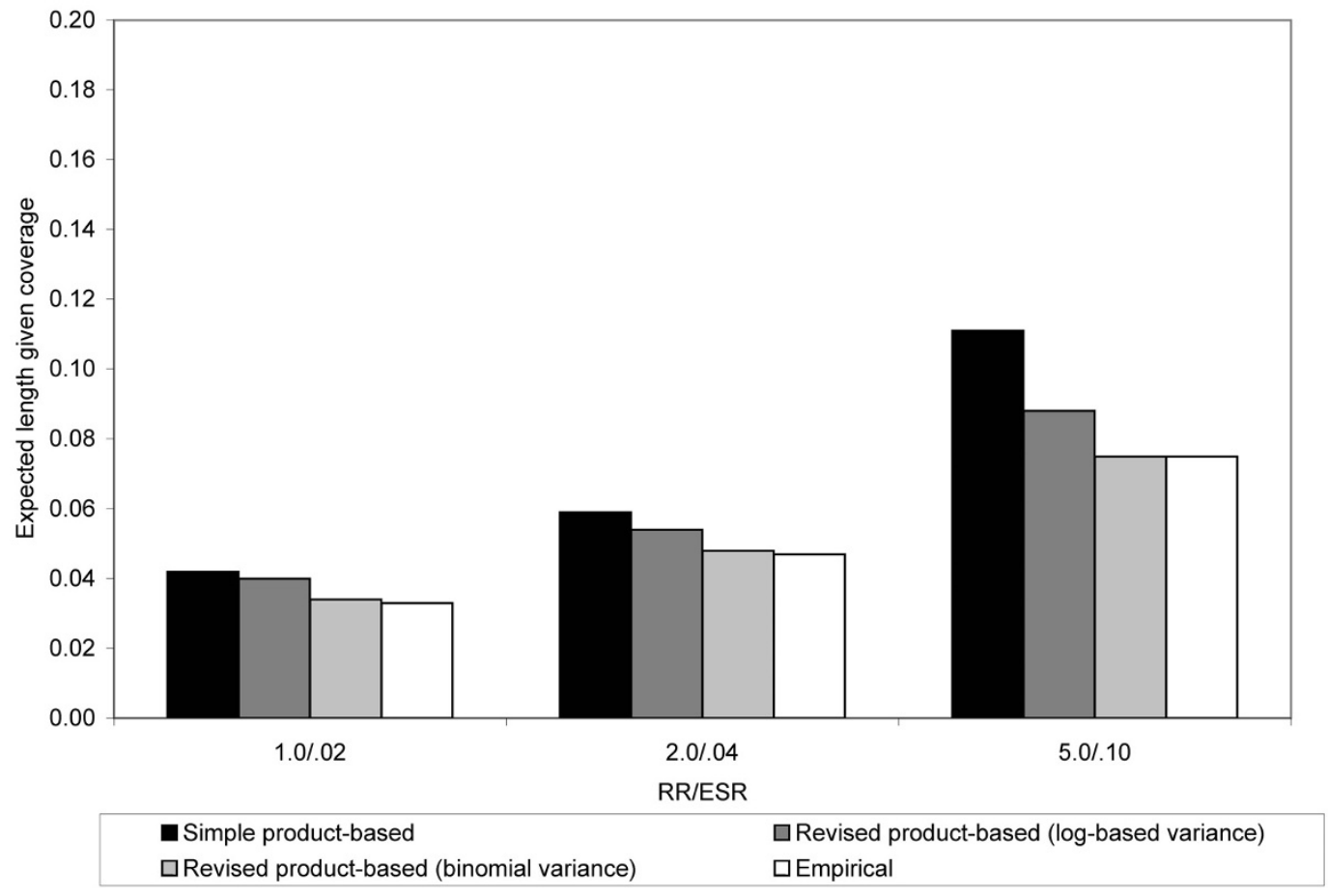

Figure 1 Expected length given coverage for $95 \%$ confidence intervals of the ESR,$E_{S} R_{R}$ using a log-based variance, and ESR ${ }_{R}$ using a binomial variance in Scenario 1. Empirical 95\% confidence intervals are also shown. The analysis assumed an exposure probability of .05 and risk of disease in the unexposed of .02. The x-axis is the magnitude of the RR. Results are from simulations where both $\mathrm{N}_{1}$ and $\mathrm{N}_{2}$ are $5,000$.

from was 1,000 and the sample size the RR was derived from was 5,000 (Table 4).

The expected lengths given coverage for Scenario 3 is shown in Figure 3. Compared to Scenario 1 (Figure 1) and Scenario 2 (Figure 2), the expected lengths have decreased substantially. Also, as in Scenario 2, the expected lengths for all of the 95\% CIs increased as the magnitude of the RR increased.

\section{Scenario 4: High exposure probability (.20)/Moderate} disease probability among unexposed (.09)

In Scenario 4 we increased both the exposure probability (.20) and the disease probability among the unexposed (.09) at the same time. This gave similar results to Scenario 3. The observed relative bias of ESR increased with increasing $R R$, while the observed relative bias was near $0 \%$ for $E_{S R}$. Coverage probabilities for the $95 \% \mathrm{CI}$ of $\mathrm{ESR}_{\mathrm{S}}$ decreased substantially as the RR increased. Coverage probabilities for the 95\% CIs for $E_{R}$ using a log-based variance and binomial variance were not affected by the magnitude of the RR. Expected lengths given coverage also showed similar relationships that were previously described (Figure 4).

In Scenario 4, we also evaluated the properties of our estimator when the sample size was 250 for both samples. We observed similar relationships in terms of observed relative bias and coverage probabilities. The observed relative bias of ESR $\mathrm{S}_{\mathrm{S}}$ was $7.8 \%$ when the RR was 1.0 and $84.9 \%$ when the $\mathrm{RR}$ was 5.0, while the observed relative bias of $\mathrm{ESR}_{\mathrm{R}}$ ranged between $-1.1 \%$ and $1.1 \%$. The coverage probability of the $95 \%$ CI for $\mathrm{ESR}_{\mathrm{S}}$ was $96.8 \%$ when the RR was 1.0 but fell below 95\% when the RR was 1.5 (92.3\%) and decreased substantially for a RR of 5.0 (56.8\%). The coverage probability of the $95 \% \mathrm{CI}$ for $\mathrm{ESR}_{\mathrm{R}}$ using a log-based variance was greater than $95 \%$ for all RRs. The coverage probability of the $95 \%$ CI for $E_{R} R_{R}$ using a binomial 


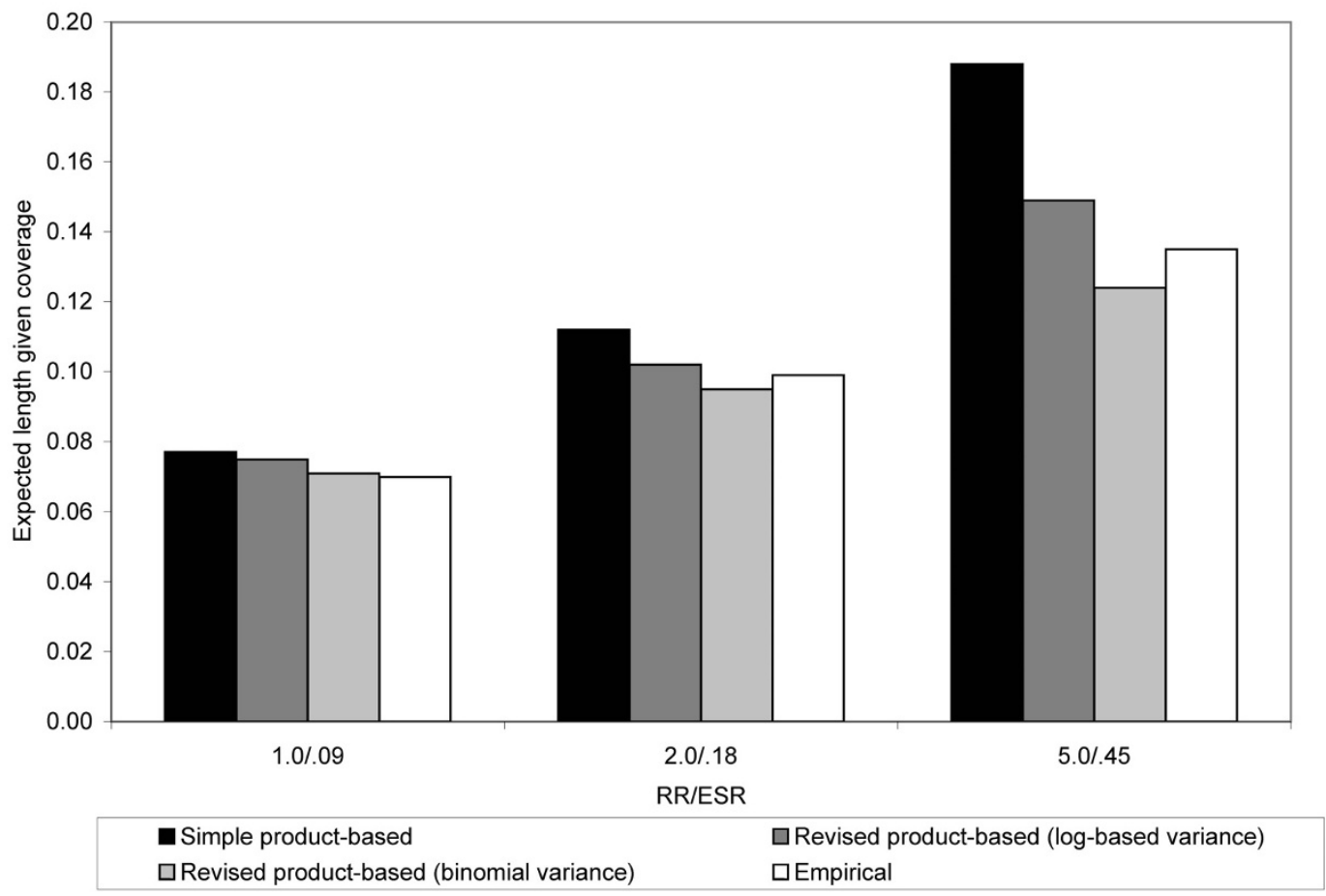

Figure 2 Expected length given coverage for $95 \%$ confidence intervals of the $E_{S R_{S}}, E R_{R}$ using a log-based variance, and $E S R_{R}$ using a binomial variance in Scenario 2. Empirical 95\% confidence intervals are also shown. The analysis assumed an exposure probability of .05 and risk of disease in the unexposed of .09. The x-axis is the magnitude of the RR. Results are from simulations where both $\mathrm{N}_{1}$ and $\mathrm{N}_{2}$ are 5,000 .

variance ranged between 85.7 and $92.5 \%$. In terms of expected length given coverage, the $95 \% \mathrm{CI}$ for $\mathrm{ESR}_{\mathrm{R}}$ using a binomial variance provided shorter intervals and were closer to the length of the empirical interval than the 95\% CI using a log-based variance.

\section{Results of the case study}

Results of the case study are shown in Table 5. The estimated risk of symptomatic knee OA was slightly higher when using the simple product-based method. The estimate of the risk of symptomatic knee OA in obese persons ranged between $0.57 \%$ and $2.11 \%$ when using the revised product-based method. All 95\% confidence intervals overlapped with one another for each age group.

\section{Discussion}

We have shown via a simulation study that the simple product-based estimator $\left(\mathrm{ESR}_{\mathrm{S}}\right)$ that has been calculated in previous studies only performs well in certain situations. Mainly, those situations are when the exposure probability is low $(\sim 5 \%)$ and the magnitude of the $R R$ is small $(\sim 3.0)$. There are two reasons for this and they can easily be seen by deconstructing the overall risk of disease using the law of total probability.

$$
P(D)=P(D \mid E) * P(E)+P(D \mid \bar{E}) * P(\bar{E})
$$

Recall that for the product-based estimator of the ESR to be unbiased that what we really need is an estimate of the risk of disease in the unexposed and not the overall risk. When the exposure probability is low, less weight is put on the probability of disease among the exposed. Put this together with a small RR and most of the overall risk of disease is being influenced by those who are unexposed. However, increasing the exposure probability puts more weight on the risk of disease 


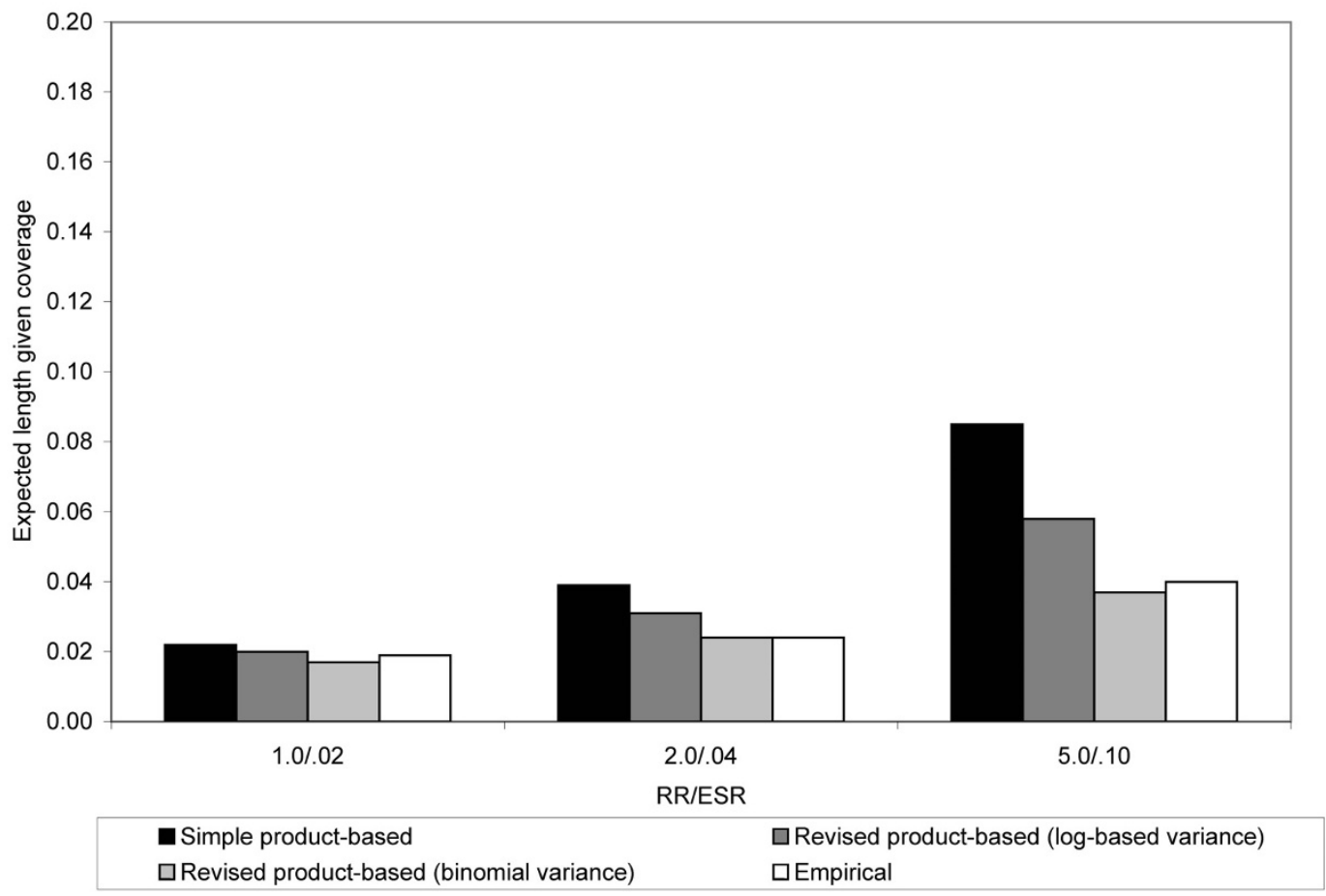

Figure 3 Expected length given coverage for $95 \%$ confidence intervals of the $E_{S R_{S}}, E R_{R}$ using a log-based variance, and ESR $R_{R}$ using a binomial variance in Scenario 3. Empirical 95\% confidence intervals are also shown. The analysis assumed an exposure probability of .20 and risk of disease in the unexposed of .02. The x-axis is the magnitude of the RR. Results are from simulations where both $\mathrm{N}_{1}$ and $\mathrm{N}_{2}$ are $5,000$.

among the exposed, which will give you a much more biased estimate of the risk of disease among the unexposed. We also showed that $\mathrm{ESR}_{\mathrm{R}}$ provides a substantial improvement over the $\mathrm{ESR}_{\mathrm{S}}$ in terms of observed relative bias. We found that the observed relative bias of $\mathrm{ESR}_{\mathrm{R}}$ was near $0 \%$ in almost all cases.

Coverage probabilities for the $95 \%$ CI for $\mathrm{ESR}_{\mathrm{S}}$ were inversely related to the observed relative bias of ESR $\mathrm{S}_{\mathrm{S}}$ As the observed relative bias increased, the coverage probability decreased. The overestimation of the ESR using existing methodology $\left(\mathrm{ESR}_{\mathrm{S}}\right)$ led to $95 \% \mathrm{CIs}$ that were less likely to cover the true ESR. Also, the expected lengths given coverage for these $95 \%$ CIs were usually longer than the lengths produced for $\mathrm{ESR}_{\mathrm{R}}$ using either the log-based variance or the binomial variance rendering this method of point and interval estimation to be sub-optimal.

Coverage probabilities for the $95 \%$ CI for $E_{R} R_{R}$ using a log-based variance exhibited greater than $95 \%$ coverage in most cases. The exception was when the sample size for the overall risk was 1,000 and the sample size for the RR was 5,000. Paradoxically, this was the only situation in which the $95 \% \mathrm{CI}$ of $\mathrm{ESR}_{\mathrm{R}}$ using a binomial variance exhibited greater than $95 \%$ coverage. In terms of expected length given coverage, neither of these two methods of interval estimation of $\mathrm{ESR}_{\mathrm{R}}$ performed better than the other in all situations. The coverage probability and expected length given coverage depended on the variance estimate that was employed. From equation 11, we can see that the log-based variance of $E_{S R}$ took into account variability from the overall risk and the RR. We also assumed that the two measures were independent and had a covariance of zero, which is a reasonable assumption because the two measures come from two independent samples. From equation 12, we can see that the binomial variance of $\mathrm{ESR}_{\mathrm{R}}$ probability of exposure from sample 2 so that the variance would not be 


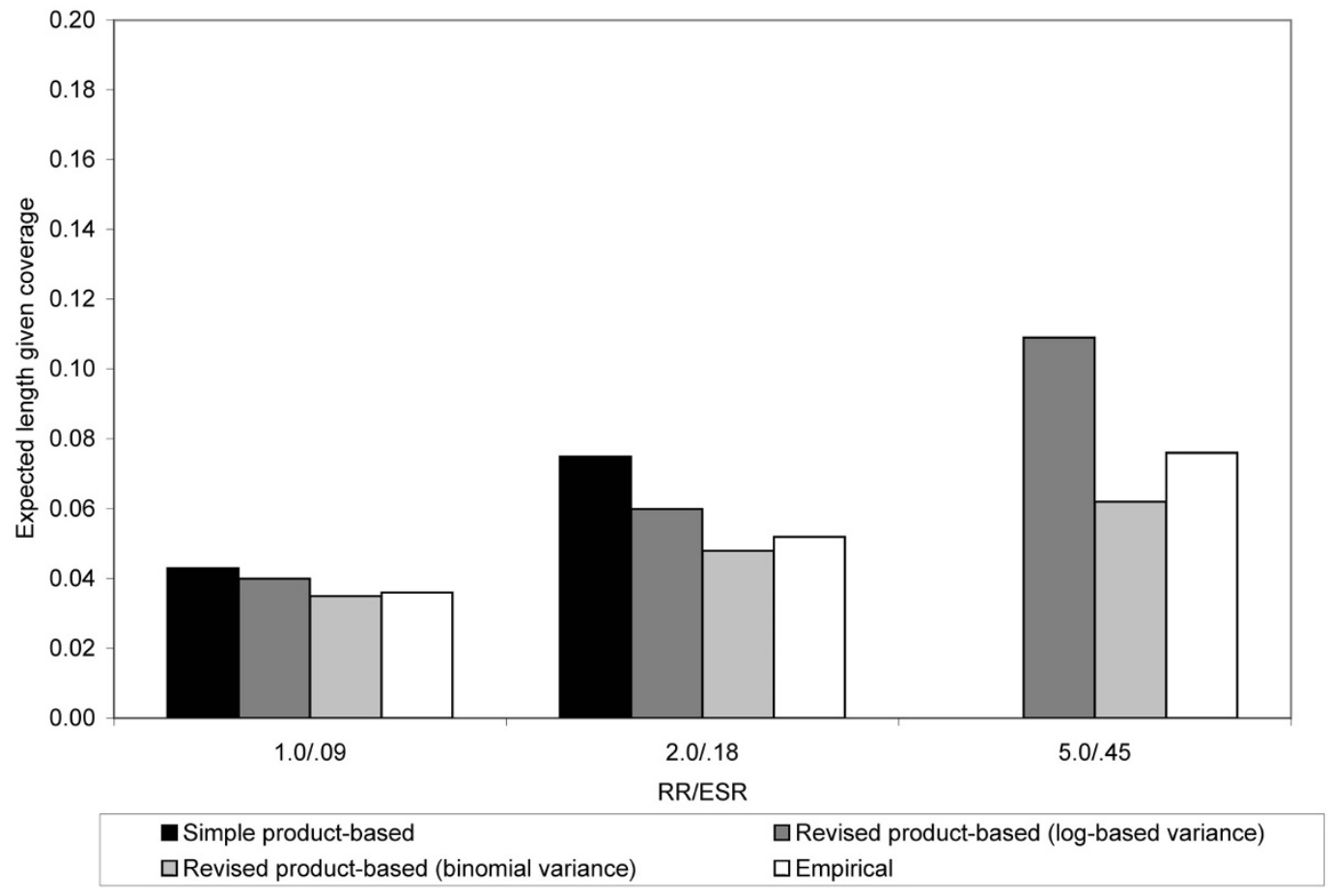

Figure 4 Expected length given coverage for $95 \%$ confidence intervals of the $E_{S R_{S}}$, ESR $R_{R}$ using a log-based variance, and $E S R_{R}$ using a binomial variance in Scenario 4. Empirical $95 \%$ confidence intervals are also shown. The analysis assumed an exposure probability of .20 and risk of disease in the unexposed of .09. The x-axis is the magnitude of the RR. Results are from simulations where both $N_{1}$ and $N_{2}$ are 5,000. Note: The expected length given coverage for the $95 \% \mathrm{Cl}$ around ESRs could not be computed when the RR was 5.0 because the coverage probability was 0 .

under-estimated. However, in most cases the variability still was under-estimated. When the sample sizes were equal, the under-estimation was very little since the coverage probabilities ranged from $87 \%-95 \%$ in most cases.
However in Scenario 1, when the sample size combination was $1,000 / 1,000$ and the RR was $1.0,1.5$, and 2.0 the coverage probabilities were $66 \%, 78 \%$, and $83 \%$ respectively.

\section{Table 5 Results from the case study on the risk of symptomatic knee OA in obese persons}

\begin{tabular}{|c|c|c|c|c|c|c|}
\hline Age & $\begin{array}{c}\text { Overall risk of } \\
\text { symptomatic knee } \\
\text { OA in the Oliveria } \\
\text { study }\end{array}$ & $\begin{array}{l}\text { Risk of symptomatic knee } O A \\
\text { for obese persons using the } \\
\text { simple product-based method }\end{array}$ & $\begin{array}{l}95 \% \mathrm{Cl} \text { for } \\
\text { ESR }_{\mathrm{S}} \text { using a } \\
\text { log-based } \\
\text { variance }\end{array}$ & $\begin{array}{l}\text { Risk of symptomatic knee } O A \\
\text { for obese persons using the } \\
\text { revised product-based } \\
\text { method }\end{array}$ & $\begin{array}{l}95 \% \mathrm{Cl} \text { for } \\
\text { ESR }_{\mathrm{R}} \text { using a } \\
\text { log-based } \\
\text { variance }\end{array}$ & $\begin{array}{l}95 \% \mathrm{Cl} \text { for } \\
\mathrm{ESR}_{\mathrm{R}} \text { using a } \\
\text { binomial } \\
\text { variance }\end{array}$ \\
\hline $\begin{array}{l}50- \\
59\end{array}$ & 0.0040 & 0.0076 & $0.0052-0.0110$ & 0.0057 & 0.0038-0.0085 & $0.0037-0.0077$ \\
\hline $\begin{array}{l}60- \\
69\end{array}$ & 0.0087 & 0.0167 & $0.0121-0.0230$ & 0.0125 & 0.0089-0.0175 & $0.0095-0.0155$ \\
\hline $\begin{array}{l}70- \\
79\end{array}$ & 0.0147 & 0.0282 & $0.0207-0.0383$ & 0.0211 & $0.0153-0.0289$ & $0.0168-0.0253$ \\
\hline
\end{tabular}

*Probability of being obese was derived from the Niu study (0.371).

**RR of symptomatic knee OA for obese persons was derived from the Niu study (1.91). 
The four scenarios, which were defined by the combinations of two different exposure probabilities (.05 and .20) and two different probabilities of disease in the unexposed (.02 and .09), did not affect the observed relative bias of $\mathrm{ESR}_{\mathrm{R}}$. However, as we increased these two parameters, the observed relative bias of ESR increased. This phenomenon was also demonstrated when comparing coverage probabilities based on the log-based variance for $\mathrm{ESR}_{\mathrm{R}}$ and $\mathrm{ESR}_{\mathrm{S}}$. When comparing coverage probabilities based on the binomial variance for $\mathrm{ESR}_{\mathrm{R}}$, the scenario does matter with larger values of the probability of exposure and/or probability of disease in the unexposed increased coverage probabilities. This is not surprising because the estimate of the binomial variance will increase with increasing exposure probabilities and increasing probability of disease among the unexposed.

Results from our case study most closely resemble scenario two where the magnitude of the $R R$ is 2.0. In scenario two, we assumed an exposure probability of 0.20 and a probability of disease in the unexposed of .02. In our case study the RR was 1.91, the exposure probability (probability of being obese) was 0.371 , and the overall risk of disease (symptomatic knee OA) ranged from 0.0087 to 0.0132 . While the simulations suggest that the estimator would be biased, the overall risk of disease is small so the difference between the two estimates in absolute terms is not large with the largest over-estimation occurring in those ages 70-79 by $0.71 \%$.

It is likely that the estimates produced by Horsburgh and Stewart et al. were accurate. In the article by Horsburgh et al on tuberculosis, he estimated the ESR of tuberculosis for those with advanced HIV infection; old, healed tuberculosis; and immunosuppressive therapy[1]. While the RR of obtaining a new case of tuberculosis is high for those with advanced HIV infection and old, healed tuberculosis, the probability of exposure is so low for these exposures that the impact of the large $R R$ would be muted. For those with immunosuppressive therapy, the RR of a new case of tuberculosis is modest (2.0) and the probability of exposure is low so the overall probability of disease is a good estimate of the probability of disease among those who are not on immunosuppressive therapy [1]. In the Stewart article, the largest $\mathrm{RR}$ is 4.62 , but this corresponds to an exposure probability of 0.001 . When the exposure probabilities are large enough to possibly impact the estimate of the ESR, the RR is low enough $(<2.0)$ to offset the possible bias [2].

An article by Cupples et al. calculated risk curves for first-degree relatives of patients with Alzheimer's disease. Their method used the odds ratio instead of the relative risk and included converting probabilities to odds [6]. Our method will allow clinicians and other researchers to find the ESR in one step, provided the summary statistics needed for the calculation $\left(P_{1}(D)\right.$, $\mathrm{RR}_{2}$, and $\left.\mathrm{P}_{2}(\mathrm{E})\right)$ are available.

We acknowledge that there are limitations with this study. The first is that simulation studies can not be considered a proof. However, we did show mathematically that the proposed estimator of the ESR is unbiased and the results of our simulation confirm this finding. It would be important to show mathematically what the true coverage probabilities are for our 95\% CIs across different RRs, exposure probabilities, and probabilities of disease among the unexposed. We also acknowledge that our simulations showed coverage probabilities that well exceed 95\% when we are calculating 95\% CIs for $\mathrm{ESR}_{\mathrm{R}}$ using a log-based variance.

We also evaluated the properties of our point and interval estimators when the sample size was small. We observed that one should only consider carrying out these calculations in smaller samples if the prevalence of exposure and disease among the unexposed is sufficiently large. If one of these values is small than the validity of the estimate of the RR may be questionable. Thus, we recommend that investigators using this methodology only use estimates that are of the highest quality.

The implications of our study are substantial. Clinicians can use these estimates to better explain risk of disease to patients. Many times clinicians and patients can misinterpret the meaning of having a certain RR of disease. Interpreting the probability of disease given a certain exposure (the ESR) is much more transparent. Future studies that examine the calculation of ESRs may look at the impact of having the odds ratio (OR) rather than the RR. Also, the consideration of under which study designs and magnitudes of the exposure/disease would an approximation using the OR be valid is an important question to answer. It is likely that the OR would be valid when the prevalence of the outcome is less than $10 \%$ but examining this rigorously would be of great importance [7]. Lastly, re-sampling and bootstrapping techniques may be a useful method of obtaining CIs with appropriate coverage.

\section{Conclusions}

We developed a new estimator for the ESR from two independent samples that exhibits more desirable properties with respect to bias and coverage than the existing methodology. The existing methodology will still perform well when the exposure probability is low. Future methodological studies should focus on the impact of ORs and re-sampling techniques. 


\section{Additional material}

Additional file 1: Tables displaying results for absolute bias, relative bias, and coverage probabilities for all simulation scenarios. Results from all simulations.

\section{List of abbreviations}

ESR: Exposure specific risk; RR: Relative risk; Cl: Confidence interval; D: Disease; D-: Without disease; E: Exposure; $E^{-}$: Without exposure; P(): Probability of; Var: Variance; Cov: Covariance; exp(): exponential function; OA: osteoarthritis.

\section{Acknowledgements}

Grant support: This research was supported in part by the National Institutes of Health, National Institute of Arthritis and Musculoskeletal and Skin Diseases grants T32 AR055885 and K24 AR057827.

\section{Author details}

${ }^{1}$ Department of Orthopedic Surgery, Brigham and Women's Hospital, 75 Francis Street, Boston, MA 02115, USA. ${ }^{2}$ Department of Biostatistics, Boston University School of Public Health, 801 Massachusetts Avenue, Boston, MA 02118, USA. ${ }^{3}$ Department of Epidemiology, Boston University School of Public Health, 715 Albany Street, Boston, MA 02118, USA. ${ }^{4}$ Massachusetts Veterans Epidemiology Research and Information Center, VA Cooperative Studies Program, Veterans Affairs Medical Center, 150 S. Huntington Ave, Jamaica Plain, MA 02130, USA.

\section{Authors' contributions}

WMR designed the simulation study, interpreted the data, wrote and critically revised the manuscript, and gave final approval of the manuscript. DG interpreted the data, critically revised the manuscript, and gave final approval of the manuscript. CRH interpreted the data, critically revised the manuscript, and gave final approval of the manuscript. EL designed the simulation study, interpreted the data, critically revised the manuscript, and gave final approval of the manuscript

\section{Competing interests}

The authors declare that they have no competing interests.

Received: 14 May 2010 Accepted: 5 January 2011

Published: 5 January 2011

\section{References}

1. Horsburgh CR Jr: Priorities for the treatment of latent tuberculosis infection in the United States. N Engl J Med 2004, 350(20):2060-2067.

2. Stewart A, Calder LD, Torgerson DJ, Seymour DG, Ritchie LD, Iglesias CP, Reid DM: Prevalence of hip fracture risk factors in women aged 70 years and over. QJM 2000, 93(10):677-680.

3. Buehler RJ: Confidence Intervals for the Product of Two Binomial Parameters. J Am Stat Assoc 1957, 52:482-93.

4. Oliveria SA, Felson DT, Reed II, Cirillo PA, Walker AM: Incidence of symptomatic hand, hip, and knee osteoarthritis among patients in a health maintenance organization. Arthritis Rheum 1995, 38(8):1134-1141.

5. Niu J, Zhang YQ, Torner J, Nevitt M, Lewis CE, Aliabadi P, Sack B, Clancy M, Sharma $L$, Felson DT: Is obesity a risk factor for progressive radiographic knee osteoarthritis? Arthritis Rheum 2009, 61(3):329-335.

6. Cupples LA, Farrer LA, Sadovnick AD, Relkin N, Whitehouse P, Green RC: Estimating risk curves for first-degree relatives of patients with Alzheimer's disease: the REVEAL study. Genet Med 2004, 6(4):192-196.

7. Zhang J, YU KF: What's the relative risk? A method of correcting the odds ratio in cohort studies of common outcomes. JAMA 1998, 280(19):1690-1691.

\section{Pre-publication history}

The pre-publication history for this paper can be accessed here: http://www.biomedcentral.com/1471-2288/11/1/prepub doi:10.1186/1471-2288-11-1

Cite this article as: Reichmann et al:: Evaluation of exposure-specific risks from two independent samples: A simulation study. BMC Medical Research Methodology 2011 11:1.

\section{Submit your next manuscript to BioMed Central and take full advantage of:}

- Convenient online submission

- Thorough peer review

- No space constraints or color figure charges

- Immediate publication on acceptance

- Inclusion in PubMed, CAS, Scopus and Google Scholar

- Research which is freely available for redistribution 